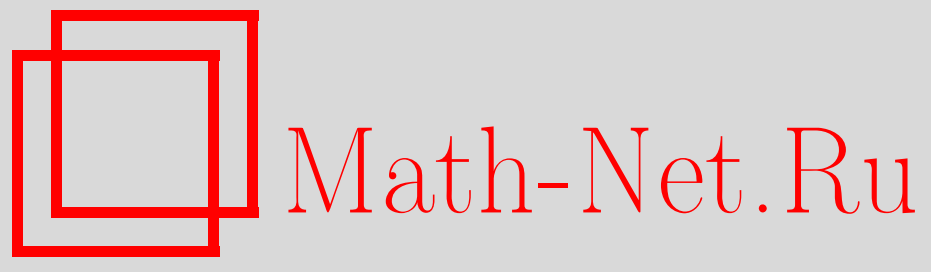

В. М. Челноков, В. Л. Зефирова, Матричный показатель маршрутной связанности узлов сети, Матем. заметки, 2009, том 85, выпуск 1, 119-130

DOI: https://doi.org/10.4213/mzm4075

Использование Общероссийского математического портала Math-Net.Ru подразумевает, что вы прочитали и согласны с пользовательским соглашением http://www . mathnet.ru/rus/agreement

Параметры загрузки:

IP : 3.85 .73 .92

26 апреля 2023 г., 14:54:32

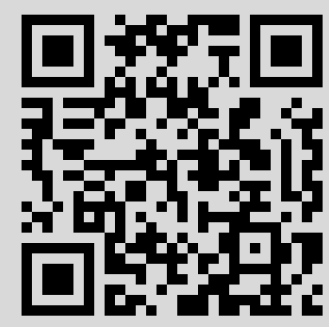


Том 85 выпуск 1 январь 2009

УДК 519.17

\section{Матричный показатель маршрутной связанности узлов сети}

\section{В. М. Челноков, В. Л. Зефирова}

Вводится показатель силы маршрутной связанности упорядоченной пары узлов сети. За основу берется вес (количество) маршрутов длины $k$, соединяющих пару, т.е. соответствующий элемент $k$-й степени сетевой матрицы, при $k \rightarrow \infty$. Применяется демпфирующий множитель $r^{-k}$, где $r$ - максимальное собственное значение сетевой матрицы. Показатель оказывается равным произведению сердцевинностей узлов пары, т.е. их координат в собственных векторах сетевой матрицы, правом и левом, соответствующих $r$. Исследуется падение в сети маршрутной связанности как результат удаления узла или связи, а именно, зависимость величины падения от структурного положения (сердцевинности) удаляемого элемента. Показывается, что критерием "ущерба" служит величина падения $r$; для нахождения этой величины применяется метод возмущений. Рассматриваются возможные приложения и числовой пример с реальной сетью большого размера (197 узлов, 780 связей).

Библиография: 15 названий.

\section{Введение}

Мы рассматриваем конечные сети, имеющие, в общем случае, направленные и взвешенные связи (дуги).

Для упорядоченных пар узлов (вершин) сети мы вводим силу связанности, базируясь на суммарном весе различных маршрутов длины $k$, соединяющих данную пару $(k \rightarrow \infty)$. Этот вес, как известно, равен соответствующему элементу $k$-й степени сетевой матрицы.

Введенная мера связанности отличается от мер аналогичного назначения, применяемых в транспортных и коммуникационных сетях. Там естественно рассматривать лишь такое сообщение между узлами, которое происходит по несамопересекающимся (простым) путям. В результате считается, что два узла связаны тем лучше (по надежности, стоимости трафика, его объему и т.п.), чем, например, меньше расстояние от одного узла до другого или чем больше число попарно независимых простых путей между этими узлами. В маршруте же, на любом по счету шаге, совершая этот шаг из любого текущего узла, можно перейти в любой узел, смежный с текущим.

Потребность в показателе маршрутной связанности возникает, поэтому, когда обращаются к цепочкам свободных ассоциаций элементов, представленных узлами сети - конкретных понятий, образов и т.п. Тогда, чтобы судить о том, насколько 
тесно связаны по смыслу два таких элемента, надо исходить, вообще говоря, изо всей совокупности маршрутов-ассоциаций между ними.

Мы также исследуем падение в сети маршрутной связанности в результате удаления узла или связи. Сравнивая сети, остающиеся после удаления различных элементов, мы выясняем зависимость эффекта удаления от структурного положения элемента в исходной сети.

В конце рассматривается числовой пример с реальной сетью большого размера (197 узлов, 780 связей).

\section{1. Обозначения}

Сеть на узлах $1,2, \ldots, n$ задается неотрицательной, квадратной матрицей $A$ порядка $n$, в которой элемент $A(i, j)$ равен весу связи $(i, j)$. В общем случае матрица не симметрична. Собственные значения $A$ и транспонированной $A^{\prime}$, как известно, одинаковы; они нумеруются в порядке невозрастания модуля: $\left|\lambda_{1}\right| \geqslant\left|\lambda_{2}\right| \geqslant\left|\lambda_{3}\right| \geqslant$ $\cdots \geqslant\left|\lambda_{n}\right|$ (каждое число $\lambda$ присутствует в этом перечне столько раз, какова алгебраическая кратность $\lambda$ ); собственные векторы-столбцы этих матриц, отвечающие $\lambda_{s}$, обозначаются $\bar{x}_{s}$ и $\bar{y}_{s}, s=1,2,3, \ldots, n$; число $r(A)=\left|\lambda_{1}\right|$ называют спектралъным радиусом $A$ (и $\left.A^{\prime}\right)$. Вектора $\bar{x}_{s}$ и $\bar{y}_{s}$ называют правым и левым собственными векторами матрицы $A$, имея в виду, что $\bar{y}_{s}^{\prime} \cdot A=\lambda_{s} \cdot \bar{y}_{s}^{\prime}$, где $\bar{y}_{s}^{\prime}$ - вектор-строка [1], $[2]$.

Элементы матрицы $A^{k}, k=1,2, \ldots$, обозначаются $A^{(k)}(i, j), i, j=1,2, \ldots, n$. Как известно, $A^{(k)}(i, j)$ есть суммарный вес различных маршрутов длиною $k$ связей, каждый из которых начинается в узле $i$, а заканчивается в узле $j$; когда вес каждой связи в сети равен 1 - это просто количество $(i, j)$-маршрутов. Маршрут из $i$ в $j-$ это последовательность связей сети такая, что началом первой связи является узел $i$, началом каждой следующей связи служит узел-конец предыдущей связи, а концом последней связи является узел $j$; при этом любой узел и любую связь маршрут может проходить любое число раз. Вес маршрута длиною $k$ связей определяется как произведение весов этих $k$ связей.

Мы будем рассматривать только сильно связные сети (просто связные, в случае ненаправленных связей); матрица такой сети называется неразложимой. Заметим, что если матрица $A$ не разложима, то и транспонированная $A^{\prime}$ не разложима, поскольку сеть $A^{\prime}$ отличается от сети $A$ лишь противоположным направлением связей.

По теореме Перрона-Фробениуса для неразложимой сетевой матрицы $A$ справедливо:

(а) ее спектральный радиус $r>0$;

(б) $r$ является собственным значением $A$, т.е. $r=\lambda_{1}$;

(в) ему отвечает положительный собственный вектор;

(г) собственное значение $r$ имеет алгебраическую кратность 1 ; отсюда вектор единственен с точностью до скалярного множителя [1].

Неразложимая, неотрицательная матрица называется примитивной, если она обладает лишь одним собственным значением, равным по модулю $r$ [1]. Примитивность $A$ гарантирует сходимость степенного метода - итерационной процедуры нахождения $r=\lambda_{1}, \bar{x}_{1}, \bar{y}_{1}$, хорошо известной в матричных вычислениях [2]-[5]. 
Собственное значение $r$ неразложимой матрицы $A$ называют перроновым корнем [1] или доминирующим собственным значением [2] матрицы $A$ (и $A^{\prime}$ ).

Координату $x_{1}(i)$ мы называем out-сердцевинностъю узла $i$, а координату $y_{1}(i)$ его in-сердиевинностью, обозначая $c_{\text {оut }}(i)=x_{1}(i), c_{\mathrm{in}}(i)=y_{1}(i), i=1,2, \ldots, n[6]$. Величина $c_{\text {out }}\left(c_{\text {in }}\right)$ коррелирует с центральностью узла: с ее увеличением возрастает вес маршрутов, исходящих из узла (приходящих в него). Кроме того, группа узлов высокой сердцевинности выделяется в сети, насколько та позволяет, повышенной плотностью - тенденцией к образованию подграфа, близкого к полному [6].

\section{2. Показатель силы связанности узлов сети}

2.1. Связанность и сердцевинность. Тесно примыкающим к показателю сердцевинности является показатель силы связанности (relatedness) пары узлов - суммарного веса маршрутов сети, соединяющих данную пару. Этот показатель, вводимый ниже, обладает свойствами, характерными для подобного рода меры [7]; а именно, по этому показателю:

- связанность пары узлов высока, если оба узла находятся в сердцевине сети (обладают высокой сердцевинностью);

- эта связанность имеет промежуточную силу, если один узел находится в сердцевине, а другой принадлежит периферии сети (обладает низкой сердцевинностью);

- она слаба, когда оба узла относятся к периферии.

Вес или количество соединяющих маршрутов ассоциируется, конечно, с элементами матрицы $A^{k}$. Однако во многих случаях они быстро растут с ростом $k$, что делает нереальным их использование напрямую. Поэтому к ним применяется компенсирующая нормировка. Сила связанности тогда вычисляется просто как произведение сердцевинностей данной пары узлов.

2.2. Произведение $c_{\text {out }} c_{\mathrm{in}}$. Действительно, пусть сетевая матрица $A$ примитивна, $r(A)=r$ и векторы $\bar{c}_{\text {out }}, \bar{c}_{\text {in }}$ пронормированы так, что скалярное произведение $\left(\bar{c}_{\text {out }}, \bar{c}_{\text {out }}\right)=\left(\bar{c}_{\text {out }}, \bar{c}_{\text {in }}\right)=1$. Тогда суммарный вес/количество $(i, j)$-маршрутов длиною $k$ связей $A^{(k)}(i, j)$ удовлетворяет соотношению

$$
\lim _{k \rightarrow \infty} r^{-k} A^{(k)}(i, j)=c_{\text {out }}(i) c_{\text {in }}(j), \quad i, j=1,2, \ldots, n
$$

[1; теорема 8.5.1]. В случае неориентированной сети этот предел равен $c(i) c(j)$, $(\bar{c}, \bar{c})=\sum c^{2}(i)=1$. Если известно только, что сеть сильно связна (просто связна, при ненаправленных связях), т.е. $A$ не разложима, то предел гарантирован лишь "в среднем":

$$
\lim _{k \rightarrow \infty} Q_{k}(i, j)=c_{\text {out }}(i) c_{\text {in }}(j), \quad \text { где } \quad Q_{k}(i, j)=\frac{1}{k} \sum_{t=1}^{k} r^{-t} A^{(t)}(i, j), \quad k=1,2, \ldots
$$

[1; теорема 8.6.1]. Заметим, что величина

$$
K(j)=\sum_{i=1}^{n} \lim _{k \rightarrow \infty} \sum_{t=1}^{k} b^{-t} A^{(t)}(i, j)
$$


напоминающая

$$
\sum_{i=1}^{n} \lim _{k \rightarrow \infty} Q_{k}(i, j) \sim c_{\mathrm{in}}(j)
$$

известна в социальных сетях как показатель Каца, измеряющий статус участника сети $(b=$ const $>r)$ [8]. Отметим также, что в [1] теоремы даны в других терминах и в этой книге вообще нет упоминаний о сердцевинности-связанности.

Именно произведение $c_{\text {out }}(i) c_{\mathrm{in}}(j)$ мы принимаем за силу связанности узла $i$ с узлом $j$. Интересна, конечно, не величина силы, сама по себе, а возможность сравнения по этой силе различных пар узлов одной и той же сети.

2.3. Случай 2-дольной сети. На самом деле, для неориентированной, связной сети мы имеем нечто большее, чем (2). Матрица $A$ здесь не примитивна тогда и только тогда, когда сеть двудольна [6] - когда множество узлов сети разбивается на две доли такие, что любые два смежных узла принадлежат разным долям. Поэтому, если сеть не является 2-дольной, то согласно (1) $\lim r^{-k} A^{(k)}(i, j)=c(i) c(j), i, j=$ $1,2, \ldots, n$. В ином случае справедлива

Теорема 1. Пусть сеть является неориентированной, связной и 2-долъной. Тогда для всякой пары узлов $i, j$, принадлежащих одной и той же доле, имеем $A^{(2 k+1)}(i, j)=0, k=1,2, \ldots, u$

$$
\lim _{k \rightarrow \infty} r^{-2 k} A^{(2 k)}(i, j)=2 c(i) c(j),
$$

а для всякой парь узлов $i, j$, принадлежащих разным долям, $A^{(2 k)}(i, j)=0, k=$ $1,2, \ldots, u$

$$
\lim _{k \rightarrow \infty} r^{-2 k-1} A^{(2 k+1)}(i, j)=2 c(i) c(j) .
$$

ДоКАЗАТЕЛЬСтво. Без потери общности полагаем совокупность узлов сети поделенной так, что $1, \ldots, n p, 0<n p<n,-$ это узлы первой доли (“" $p$-узлы"), a $n p+1$, $\ldots, n p+n t=n-$ узлы второй доли ("t-узлы"). Матрица $A$ тогда имеет нулевые квадратные подматрицы - порядка $n p$ в левом верхнем углу, и порядка $n t$ в правом нижнем. В силу такой структуры $A$ получаем

$$
\begin{aligned}
& A^{(2 k+1)}(i, j)=0, \quad k=1,2, \ldots, \quad \text { когда } \quad 1 \leqslant i, j \leqslant n p \text { или } n p+1 \leqslant i, j \leqslant n, \\
& A^{(2 k)}(i, j)=0, \quad k=1,2, \ldots, \quad \text { когда } \quad 1 \leqslant i \leqslant n p, n p+1 \leqslant j \leqslant n, \\
& \text { или } n p+1 \leqslant i \leqslant n, \quad 1 \leqslant j \leqslant n p .
\end{aligned}
$$

Далее, матрицы $A^{2 k}, k=1,2, \ldots$, имеют ненулевые элементы лишь в угловых квадратных подматрицах $P^{k}$ и $T^{k}$, расположенных на месте нулевых подматриц матрицы $A$. Матрица $P$ примитивна, так как ее сеть (на $p$-узлах) связна и однодольна [6]. Аналогичным образом примитивна $T$, задающая сеть на $t$-узлах. Эти две матрицы имеют перронов корень $r^{2}(A)=r^{2}$ и векторы $\bar{c}(P)=\overline{c p}, \bar{c}(T)=\overline{c t}$, координаты которых образуют 2-дольное разбиение координат вектора $\bar{c}(A)=\bar{c}$. Точнее, $\overline{c p}$ и $\overline{c t}$ образуют разбиение вектора $\sqrt{2} \bar{c}$, где множитель $\sqrt{2}$ возникает, если эвклидовы длины $\overline{c p}, \overline{c t}$ и $\bar{c}$ приведены к 1 .

Применяя (1) к матрицам $P$ и $T$, получаем формулу (3). С помощью (3) получаем формулу (4):

$$
\lim _{k \rightarrow \infty} r^{-2 k-1} A^{(2 k+1)}(i, j)=r^{-1} \sum_{q=1}^{n} A(i, q) \lim _{k \rightarrow \infty} r^{-2 k} A^{(2 k)}(q, j)
$$




$$
=2 r^{-1} c(j) \sum_{q=1}^{n} A(i, q) c(q)=2 c(i) c(j) .
$$

Теорема доказана.

2.4. Применение в системе текстового поиска. Содержание хранимой совокупности документов представляется в системе множеством индексирующих терминов, так что возникает 2-дольная структура “документы-термины". Значение $A(i, j)$ равно весу термина $t_{j}$ в документе $p_{i}$ (в простейшем случае 1 или 0 ). Поисковый запрос обрабатывается как дополнительный документ $q$; он временно включается в структуру, тоже подвергаясь индексированию. Система измеряет связанность по смыслу “документа" $q$ и каждого из документов $p_{i}$. Пользователю выдается ранжировка $p_{i}$ по этому критерию. В качестве критерия применяются коэффициенты косинуса, Дайса, Жаккара и др. [9]. Все они пропорциональны $A^{(2)}\left(q, p_{i}\right)$. Усовершенствованием будет замена этой величины $A^{(2)}$ величиной $A^{(k)}\left(q, p_{i}\right)$, точнее, $c(q) c\left(p_{i}\right)$ (или $c(q) c\left(p_{i}\right) A^{(2)}\left(q, p_{i}\right)$, как в некоторых когнитивно-психологических моделях, имеющих экспериментальное подтверждение [10]).

В статье [7] описано применение показателя $c(i) c(j)$ для выявления сердцевинно-периферийной структуры социальных сетей и сетей цитирования. Статья [8] содержит обзор показателей статуса узла в этих сетях; из этого обзора также можно увидеть прикладной смысл нашего показателя.

\section{3. Сравнение сетей по маршрутной связанности}

Выше мы ввели критерий сравнения по маршрутной связанности пар узлов одной и той же сети. Перейдем теперь к сравнению сетей.

Маршрутную связанность сети естественно измерять некоторым весом матрицы $A^{k}, k \rightarrow \infty$, поскольку ее элемент $A^{(k)}(i, j)$ равен весу/числу маршрутов сети от узла $i$ к узлу $j$ длиною $k$ связей. За вес $A^{k}$ можно принять, например, $S\left(A^{k}\right)=\sum_{i, j=1}^{n} A^{(k)}(i, j)$. Поскольку элементы $A^{k}$ могут неограниченно возрастать с ростом $k$, прибегнем к асимптотическому сравнению.

Обозначим через $V$ множество узлов сети. Греческими буквами $\alpha, \beta, \gamma, \ldots$ будем обозначать непустые подмножества множества $V \times V$ всех различных упорядоченных пар узлов сети. Обозначим также

$$
S(\gamma, k)=\sum_{(i, j) \in \gamma} A^{(k)}(i, j)
$$

в частности, $S(V \times V, k)=S\left(A^{k}\right)$.

Теорема 2. Пусть имеются две сети на множествах узлов $V_{1}$ и $V_{2}$, с матрицами $A_{1}$ и $A_{2}$. Пусть $A_{1}$ и $A_{2}$ примитивны, $r\left(A_{1}\right)=r_{1}, r\left(A_{2}\right)=r_{2}$. Тогда при любых $\alpha, \beta$ таких, что $\alpha \in V_{1} \times V_{1}, \beta \in V_{2} \times V_{2}$, величина $S_{1}(\alpha, k) / S_{2}(\beta, k)$ при $k \rightarrow \infty$ асимптотически пропорциональна величине $\left(r_{1} / r_{2}\right)^{k}$, т.е. отночение данных величин есть в пределе конечное положительное число.

ДокАЗАТЕЛЬСтво. Из формулы (1)

$$
\lim r^{-k} S(\gamma, k)=\sum_{i, j \in \gamma} \lim r^{-k} A^{(k)}(i, j)=\sum_{i, j \in \gamma} c_{\text {out }}(i) c_{\mathrm{in}}(j) .
$$


Поэтому

$$
\lim _{k \rightarrow \infty} \frac{S_{1}(\alpha, k)}{S_{2}(\beta, k)}\left(\frac{r_{1}}{r_{2}}\right)^{-k}=\frac{\lim r_{1}^{-k} S_{1}(\alpha, k)}{\lim r_{2}^{-k} S_{2}(\beta, k)}=s,
$$

где $0<s<\infty$. Теорема доказана.

ТЕОрема 3. Утверждение теоремы 2 справедливо для неориентированных, связных сетей, не являюшихся 2-дольными.

ДокАЗАТЕЛЬСТво следует из того, что матрицы таких сетей примитивны [6].

Теорема 4. Пусть имеются две неориентированные, связные, 2-дольные сети на множествах узлов $V_{1}$ и $V_{2}$ с матрицами $A_{1} u A_{2}, r\left(A_{1}\right)=r_{1}, r\left(A_{2}\right)=r_{2}$. Пусть $\alpha \in V_{1} \times V_{1}, \beta \in V_{2} \times V_{2}$. Тогда при $k \rightarrow \infty$ справедливо:

(а) если в каждой паре узлов из $\alpha$ и $\beta$ оба узла принадлежат одной и той же доле сети, то отношение $S_{1}(\alpha, 2 k) / S_{2}(\beta, 2 k)$ асимптотически пропориионально $\left(r_{1} / r_{2}\right)^{2 k}$

(б) если каждую пару из $\alpha$ и $\beta$ образуют узль, принадлежащие разным долям, то отношение $S_{1}(\alpha, 2 k+1) / S_{2}(\beta, 2 k+1)$ асимптотически пропориионально $\left(r_{1} / r_{2}\right)^{2 k+1}$.

ДокАЗАТЕЛЬСТво получаем из формул (3) и (4) аналогично доказательству теоремы 2.

Теорема 5. Пусть имеются две сети, матрииы которых $A_{1}$ и $A_{2}$ не разложимы, $r\left(A_{1}\right)=r_{1}, r\left(A_{2}\right)=r_{2}$, т.е. обе сети сильно свлзны (просто связны, в случае ненаправленных связей). Тогда при $k=1,2, \ldots$

$$
\frac{a}{b}\left(\frac{r_{1}}{r_{2}}\right)^{k} \leqslant \frac{S\left(A_{1}^{k}\right)}{S\left(A_{2}^{k}\right)} \leqslant a b\left(\frac{r_{1}}{r_{2}}\right)^{k},
$$

где числа $а$ и $b$ не зависят от $k, a>0, b \geqslant 1$.

ДокАЗАТЕЛЬСТво следует из того, что для всякой неразложимой сетевой матрицы $A$ порядка $n$ справедливо

$$
\frac{r^{k}}{f} \leqslant \sum_{j=1}^{n} A^{(k)}(i, j) \leqslant r^{k} f
$$

где $r=r(A), f=\max _{1 \leqslant i \leqslant n} c_{\text {out }}(i) / \min _{1 \leqslant i \leqslant n} c_{\text {out }}(i), k=1,2, \ldots$ [1; следствие 8.1.33].

Таким образом, для сравнения двух сетей достаточно сравнить $r_{1}$ и $r_{2}$.

Величина $S\left(A^{k}\right)$ есть матричная норма матрицы $A^{k}$, называемая $l_{1}$-нормой [1]. Матричной нормой является также $n M\left(A^{k}\right)$, где $M=\max _{i, j} A^{(k)}(i, j)$ [1]. Упомянем также максимальную столбцовую норму $\|\cdot\|_{1}$ и максимальную строчную норму $\|\cdot\|_{\infty}$, определяемые для неотрицательной матрицы как ее максимальная столбцовая/строчная сумма [1].

Любая матричная норма измеряет "вес", "величину" (“магнитуду") матрицы, отражающую величину ее элементов; как функция на множестве матриц, она удовлетворяет некоторому стандартному набору аксиом [1]. В конечномерном пространстве любые две матричные нормы $\|\cdot\|_{\text {I }}$ и $\|\cdot\|_{\text {II }}$ эквивалентны друг другу: существуют 
положительные константы $g$ и $h$ такие, что при любой $(n \times n)$-матрице $A$ справедливо $g\|A\|_{\text {II }} \leqslant\|A\|_{\mathrm{I}} \leqslant h\|A\|_{\text {II }}$ (т.е. $g$ и $h$ зависят от этих двух норм и $n$ и не зависят от $A)$ [1]. Поэтому если $A_{1}$ и $A_{2}$ - две любые $(n \times n)$-матрицы и $\|\cdot\|$ - любая матричная норма, то из эквивалентности этой нормы и $l_{1}$-нормы

$$
\frac{g}{h} \leqslant \frac{S\left(A_{1}^{k}\right)}{S\left(A_{2}^{k}\right)}\left(\frac{\left\|A_{1}^{k}\right\|}{\left\|A_{2}^{k}\right\|}\right)^{-1} \leqslant \frac{h}{g} .
$$

Для справки, $h / g=n^{2}$, когда $\|\cdot\|$ есть $n M$, и $h / g=n$, когда $\|\cdot\|$ есть $\|\cdot\|_{1}$ или $\|\cdot\|_{\infty}[1]$.

\section{4. Чувствительность сети к удалениям узлов и связей}

Ниже мы исследуем падение в сети маршрутной связанности после удаления одного узла или одной связи. Сравнивая маршрутную связанность в сетях, остающихся после удаления, мы выясняем, как зависит "ущерб” от структурного положения удаляемого элемента в исходной сети.

Мы сравниваем лишь сети, которые сильно связны (просто связны в случае ненаправленных связей), т.е. сети, матрицы которых неразложимы. Тогда по суммарному весу маршрутов длины $k$ одна сеть неограниченно превосходит другую при $k \rightarrow \infty$, если перронов корень $r$ у первой сети больше, чем у второй (теорема 5 ). Если же, вдобавок, матрицы сетей примитивны или симметричны, то не только первая сеть превосходит по маршрутам вторую на уровне сети в целом, но и любой набор пар узлов из первой сети (в частности, любая одна пара) превосходит по маршрутам, соединяющим эти пары, любой набор пар из второй сети (теоремы 2-4).

Поскольку мы сравниваем сети с одинаковым числом узлов, сравнение по суммарной связанности всех пар узлов $\left(S\left(A^{k}\right), k \rightarrow \infty\right)$ равносильно сравнению по средней связанности одной пары.

4.1. Эффект удаления узла или связи. Таким образом, критерием сравнения сетей, получаемых в результате удалений из данной сети, служит величина падения $r$. Эта величина у нас всегда положительна. Действительно, при удалениях узлов мы сравниваем сети, матрицы которых получаются из матрицы $A$ исходной сети вычеркиванием соответствующей строки и столбца; так как по условию $A$ неразложима, перронов корень каждой из получаемых матриц меньше $r=r(A)$ [11; теорема 0.6]. Матрицы сетей, остающихся после удаления связей, имеют вид $\widetilde{A}=A-Z$, где $Z$ - некоторая ненулевая, неотрицательная матрица; по условию $\widetilde{A}$ неразложима, поэтому $r(\widetilde{A})<r[1 ;$ с. 607], [11; теорема 0.7], [12; 18.11].

4.2. Эффект как функция сердцевинности. Величину $\widetilde{r}=r(\widetilde{A})$ можно вычислить, рассматривая матрицу $Z$ как возмущение матрицы $A$ (когда удаляется узел $i, \widetilde{A}$ получается из $A$ обнулением $i$-х строки и столбца; сеть, которая остается, имеет перронов корень, равный $\widetilde{r})$.

Для вычисления $\widetilde{r}^{(1)}$, первого приближения $\widetilde{r}$, воспользуемся формулой из $[1$; c. 444]; по этой формуле в наших обозначениях при $\left(\bar{c}_{\text {out }}, \bar{c}_{\text {out }}\right)=\left(\bar{c}_{\text {out }}, \bar{c}_{\text {in }}\right)=1$,

$$
\frac{\partial r}{\partial A(i, j)}=c_{\text {out }}(j) c_{\text {in }}(i) .
$$


Отсюда

$$
\Delta r \approx \sum_{i, j} \frac{\partial r}{\partial A(i, j)} \Delta A(i, j)=\sum_{i, j} c_{\mathrm{out}}(j) c_{\mathrm{in}}(i) \Delta A(i, j) .
$$

При удалении произвольного множества связей $L$ имеем $\Delta A(i, j)=-A(i, j),(i, j) \in L$, и получаем общую формулу

$$
r-\widetilde{r}^{(1)}=\sum_{(i, j) \in L} A(i, j) c_{\mathrm{out}}(j) c_{\mathrm{in}}(i) .
$$

Удаление узла $v$ означает ликвидацию всех связей типа $(v, i),(i, v)$; для этого случая (5) дает

$$
r-\widetilde{r}^{(1)}(v)=c_{\mathrm{in}}(v) \sum_{i} A(v, i) c_{\mathrm{out}}(i)+c_{\mathrm{out}}(v) \sum_{i} A(i, v) c_{\mathrm{in}}(i)=2 r c_{\mathrm{out}}(v) c_{\mathrm{in}}(v) ;
$$

для неориентированной сети $A(i, v)=A(v, i), \bar{c}_{\text {out }}=\bar{c}_{\text {in }}=\bar{c},(\bar{c}, \bar{c})=1$ и, следовательно,

$$
r-\widetilde{r}^{(1)}(v)=2 r c^{2}(v) .
$$

При удалении направленной связи $(v, i)$ согласно (5)

$$
r-\widetilde{r}^{(1)}(v, i)=A(v, i) c_{\mathrm{out}}(i) c_{\mathrm{in}}(v) .
$$

В случае же неориентированной сети

$$
r-\widetilde{r}^{(1)}(v, i)=A(v, i) c_{\mathrm{out}}(i) c_{\mathrm{in}}(v)+A(i, v) c_{\mathrm{out}}(v) c_{\mathrm{in}}(i)=2 A(v, i) c(v) c(i) .
$$

Таким образом, как показывают формулы (6)-(9), пунктами наибольшей уязвимости сети являются в первом приближении узлы максимальной сердцевинности. Точность приближения тем выше, чем меньше $\|Z\| /\|A\|$.

4.3. Второе приближение. Стандартные результаты теории возмущений [2], $[12 ; 16.50],[4],[5]$ в нашем случае принимают вид

$$
\widetilde{r}^{(1)}=r-\left(Z \bar{c}_{\text {out }}, \bar{c}_{\text {in }}\right),
$$

и во втором приближении

$$
\widetilde{r}^{(2)}=\widetilde{r}^{(1)}+\sum_{s=2}^{n} \frac{\left(Z \bar{c}_{\text {out }}, \bar{y}_{s}\right)\left(Z \bar{x}_{s}, \bar{c}_{\text {in }}\right)}{r-\lambda_{s}}
$$

при нормировке $\left(\bar{x}_{s}, \bar{x}_{s}\right)=\left(\bar{x}_{s}, \bar{y}_{s}\right)=1$; для неориентированной сети $\bar{c}_{\text {out }}=\bar{c}_{\text {in }}=\bar{c}$, $\bar{y}_{s}=\bar{x}_{s}$ и

$$
\widetilde{r}^{(1)}=r-(Z \bar{c}, \bar{c}), \quad \widetilde{r}^{(2)}=\widetilde{r}^{(1)}+\sum_{s=2}^{n} \frac{\left(Z \bar{c}, \bar{x}_{s}\right)^{2}}{r-\lambda_{s}} .
$$

При удалении узла $v$ матрица $Z$ получается из матрицы $A$ сохранением строки и столбца, имеющих номер $v$, и обнулением всех остальных строк и столбцов. Для такой матрицы $Z$ находим

$$
\begin{gathered}
\left(Z \bar{c}_{\text {out }}, \bar{c}_{\mathrm{in}}\right)=2 r c_{\mathrm{out}}(v) c_{\mathrm{in}}(v) \quad(\text { совпадает с }(6)), \\
\left(Z \bar{c}_{\mathrm{out}}, \bar{y}_{s}\right)=\left(r+\lambda_{s}\right) c_{\mathrm{out}}(v) y_{s}(v), \quad\left(Z \bar{x}_{s}, \bar{c}_{\mathrm{in}}\right)=\left(r+\lambda_{s}\right) x_{s}(v) c_{\mathrm{in}}(v) ;
\end{gathered}
$$


подставив это в (10), получаем

$$
r-\widetilde{r}^{(2)}(v)=c_{\text {out }}(v) c_{\text {in }}(v)\left[2 r-\sum_{s=2}^{n} \frac{\left(r+\lambda_{s}\right)^{2} x_{s}(v) y_{s}(v)}{r-\lambda_{s}}\right]
$$

для неориентированной сети

$$
r-\widetilde{r}^{(2)}(v)=c^{2}(v)\left[2 r-\sum_{s=2}^{n} \frac{\left(r+\lambda_{s}\right)^{2} x_{s}^{2}(v)}{r-\lambda_{s}}\right]
$$

Когда удаляется направленная связь $(v, i)$, в матрице $Z$ все элементы равны нулю, кроме одного: $Z(v, i)=A(v, i)$. Тогда по формуле $(10)$

$$
r-\widetilde{r}^{(2)}(v, i)=A(v, i) c_{\mathrm{out}}(i) c_{\mathrm{in}}(v)\left[1-A(v, i) \sum_{s=2}^{n} \frac{x_{s}(i) y_{s}(v)}{r-\lambda_{s}}\right] .
$$

Когда же сеть неориентированная и удаляется связь $(v, i)$, все элементы $Z$ равны нулю, за исключением двух: $Z(v, i)=Z(i, v)=A(v, i)$. В этом случае

$$
r-\widetilde{r}^{(2)}(v, i)=2 A(v, i) c(v) c(i)-A^{2}(v, i) \sum_{s=2}^{n} \frac{\left[c(v) x_{s}(i)+c(i) x_{s}(v)\right]^{2}}{r-\lambda_{s}} .
$$

\section{5. Числовой пример}

Мы провели вычисления на компьютере для сети взаимных гипертекстовых ссылок словаря терминов линейной алгебры (Linear Algebra Glossary, Google's Directory, www.google.com). Полное количество узлов-терминов равнялось 201. Для большей простоты мы сделали связи-ссылки ненаправленными. Вес каждой связи был равен 1. Компьютер выделил из полученного графа максимальную связную компоненту - неориентированный граф, который мы и рассматриваем ниже. Его основные данные: число узлов $n=197$, число связей $m=780$, средняя степень $2 m / n=7.9$, максимальная степень - 54, минимальная - 1 .

Матрица смежности симметрична, поэтому все собственные значения вещественны. Максимальное среди них $r=\lambda_{1}=14.3196$, остальные, по убыванию модуля, равны: ${ }^{1}$

\begin{tabular}{|c|c|c|c|c|c|c|c|c|c|c|}
\hline$\lambda_{2}$ & $\lambda_{3}$ & $\lambda_{4}$ & $\lambda_{5}$ & $\lambda_{6}$ & $\lambda_{7}$ & $\lambda_{8}$ & $\lambda_{9}$ & $\ldots$ & $\lambda_{196}$ & $\lambda_{197}$ \\
\hline 8.43 & 7.44 & -7.15 & -6.75 & 6.51 & 6.45 & -6.43 & 5.97 & $\ldots$ & 0.004 & 0.0009 \\
\hline
\end{tabular}

Полученные 197 значений сердцевинности в ранжировке по убыванию приводятся в табл. 1 . Обозначения: $d(i)-$ степень узла $i$.

Более половины узлов (99/197) имеют сердцевинность лишь $11 \%$ или меньше от максимальной, сердцевинностью выше $50 \%$ от максимальной обладает группа, содержащая только $3.5 \%$ (7/197) узлов. Степень выше $44 \%(24 / 54)$ от максимальной

\footnotetext{
13начение $r$ и вектор $\bar{c}$ были найдены степенным методом, а значения $\lambda_{s}$ и векторы $\bar{x}_{s}-$ методом исчерпывания (дефляции), который состоит в применении степенного метода к некоторым образом редуцированной матрице смежности [3].
} 
ТАБЛИЦА 1

\begin{tabular}{|c|c|c|c|c|}
\hline № $\Pi / \Pi$ & $i$ & $c(i) / c_{\max }$ & $d(i)$ & Термин \\
\hline 1 & 46 & 1 & 54 & собственные значения \\
\hline 2 & 185 & 0.76 & 40 & симметричная матрица \\
\hline 3 & 84 & 0.64 & 31 & обратная матрица \\
\hline 4 & 47 & 0.56 & 26 & собственные векторь \\
\hline 5 & 146 & 0.55 & 28 & положительно определенная матрица \\
\hline 6 & 134 & 0.54 & 24 & ортогональная матрица \\
\hline 7 & 190 & 0.52 & 20 & транспонированная матрица \\
\hline 8 & 168 & 0.49 & 22 & вырожденная матрища \\
\hline$\ldots$ & .. & $\cdots$ & .. & $\ldots \ldots \ldots \ldots \ldots \ldots \ldots$ \\
\hline 197 & 3 & 0.003 & 1 & присоединенная (adjoint) матрица \\
\hline
\end{tabular}

имеют лишь $3.5 \%$ узлов, тогда как у $53 \%$ (105/197) из них она меньше $11 \%(6 / 54)$ от максимальной; см. также [6].

В табл. $2^{2}$ приводятся реальные величины $\widetilde{r}(v)$ для графа, который получается из исходного графа в результате удаления узла $v$. В этой таблице $d(v)-$ степень $v$, $c(v)$ - сердцевинность $v, \widetilde{r}^{(1)}(v)$ и $\widetilde{r}^{(2)}(v)$ - первое и второе приближения $\widetilde{r}(v)$, по формулам теории возмущений (7) и (11). Узлы $v$ следуют в порядке убывания $c(v)$.

ТАБЛИЦА 2

\begin{tabular}{|c|c|c|c|c|c|c|c|}
\hline № п/п & 1 & 2 & 3 & 4 & 5 & $\ldots$ & 62 \\
\hline \hline$v$ & 46 & 185 & 84 & 47 & 146 & $\ldots$ & 153 \\
\hline$d(v)$ & 54 & 40 & 31 & 26 & 28 & $\ldots$ & 8 \\
\hline$c(v)$ & 0.335 & 0.253 & 0.215 & 0.187 & 0.185 & $\ldots$ & 0.05617 \\
\hline$\widetilde{r}(v)$ & 12.88 & 13.49 & 13.70 & 13.86 & 13.87 & $\ldots$ & 14.2773 \\
\hline$\widetilde{r}^{(1)}(v)$ & 11.11 & 12.48 & 12.99 & 13.31 & 13.33 & $\ldots$ & 14.2292 \\
\hline$\widetilde{r}^{(2)}(v)$ & 13.17 & 13.45 & 13.84 & 14.00 & 14.02 & $\ldots$ & 14.2812 \\
\hline
\end{tabular}

На рис. 1 показаны графики зависимости от $c(v)$ величин $r-\widetilde{r}(v), r-\widetilde{r}^{(1)}(v)$ и $r-\widetilde{r}^{(2)}(v)$, построенные по табл. 2. Обозначения: delta $=(r-\widetilde{r}) \times 100$, delta1 $=$ $\left(r-\widetilde{r}^{(1)}\right) \times 100$, delta2 $=\left(r-\widetilde{r}^{(2)}\right) \times 100$.

\section{Заключение}

Падение маршрутной связанности в сети после удаления узла оказывается, в первом приближении, тем значительней, чем больше произведение out- и in-cepдцевинности этого узла; в неориентированной сети оно пропорционально квадрату сердцевинности. Такого же рода зависимость имеет место и при удалении связи.

В то же время, по нашим наблюдениям, в больших реальных сетях в сердцевину (группу узлов наибольшей сердцевинности) часто входит очень малая доля узлов; это может выражаться, например, в том, что лишь несколько процентов от общего

\footnotetext{
${ }^{2}$ Полные данные можно получить у авторов.
} 

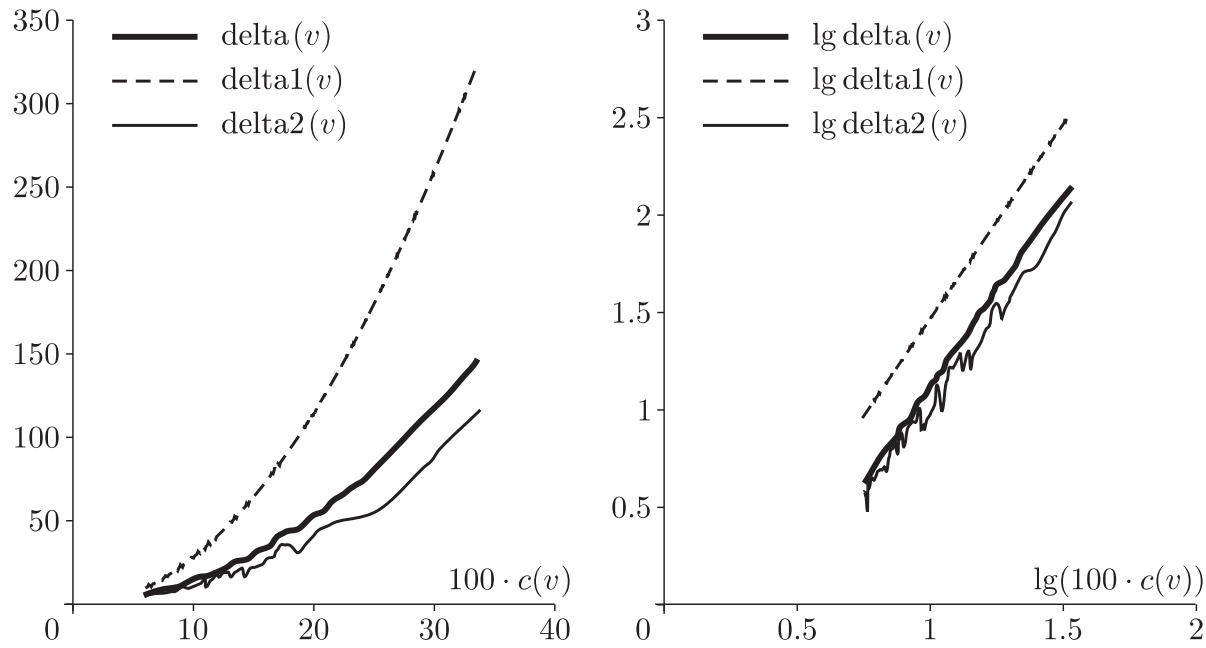

Рис. 1. Реальная и приближенная величина падения связанности сети

числа узлов сети обладает сердцевинностью выше $50 \%$ от максимальной, тогда как сердцевинность остальных узлов ниже этого.

В результате, сердцевина образует в сети зону уязвимости - наибольшего потенциального ущерба, однако, при случайных, равновероятных удалениях узлов большой “ущерб" маловероятен, так как удаляемый узел будет, скорее всего, относиться к узлам за пределами сердцевины в силу их преобладания.

Здесь имеется некоторая аналогия с результатами исследований структурной устойчивости (робастности) Интернет, Всемирной Паутины и других больших сетей к отключениям узлов [13], [14]. Ущерб измерялся там величиной возрастания среднего расстояния между парой узлов. Если центральные узлы (с высокой степенью числом связей) образуют в сети малую группу, то сеть очень чувствительна по этому показателю к их выпадению. С другой стороны, эмпирически, многие большие реальные сети почти не чувствительны к случайным отключениям узлов. Оказывается, в этих сетях число связей узла имеет степенное распределение, при котором как раз и возникает упомянутая группа. Поэтому случайные отказы узлов приходятся в основном на малостепенные узлы. Центральным же узлам угрожают специально организованные (хакерские) атаки.

Что касается Всемирной Паутины, то для нее более подходящим критерием ущерба, как мы полагаем, является падение маршрутной связанности, поскольку в этой сети для перемещения пользователя между узлами (вэб-страницами) по гипертекстовым связям характерны траектории типа маршрутов. Об этом говорит успешное применение модели случайного блуждания для определения рейтинга вэб-страниц поисковой системой Google [8], [15]. Рейтингом служит, в наших терминах, вектор in-сердцевинностей стохастической сетевой матрицы [6].

\section{СПИСОК ЦИТИРОВАННОЙ ЛИТЕРАТУРЫ}

[1] Р. Хорн, Ч. Джонсон, Матричный анализ, Мир, М., 1989.

[2] Дж. Х. Голуб, Ч.Ф. Ван Лоун, Матричные вычисления, Мир, М., 1999. 
[3] Д. К. Фаддеев, В. Н. Фаддеева, Вычислительные методы линейной алгебры, Физматгиз, М., 1963.

[4] Дж. Х. Уилкинсон, Алгебраическая проблема собственных значений, Наука, М., 1970.

[5] И. М. Гельфанд, Лекиии по линейной алгебре, Наука, М., 1966.

[6] В. М. Челноков, В.Л. Зефирова, "Матричный показатель степени принадлежности узла сети сердцевине сети", Матем. заметки, 80:6 (2006), 908-919.

[7] S. P. Borgatti, M. G. Everett, "Models of core/periphery structures", Social Networks, 21:4 (2000), 375-395.

[8] J. M. Kleinberg, "Authoritative sources in a hyperlinked environment", J. ACM, 46:5 (1999), 604-632.

[9] G. Salton, Automatic text processing, Addison-Wesley, Reading, MA, 1989.

[10] W. Kintsch, D. M. Welsch, "The construction-integration model: a framework for studying memory for text", Relating Theory and Data: Essays on Human Memory, Erlbaum, Hillsdale, NJ, 1991, 367-386.

[11] Д. Цветкович, М. Дуб, Х. Захс, Спектры графов: Теория и применение, Наукова думка, Киев, 1984.

[12] В. В. Воеводин, Ю. А. Кузнецов, Матрицы и вычисления, Наука, М., 1984.

[13] R. Albert, H. Jeong, A.-L. Barabási, "Error and attack tolerance of complex networks", Nature, 406 (2000), 378-382.

[14] R. Albert, A.-L. Barabási, "Statistical mechanics of complex networks", Rev. Modern Phys., 74:1 (2002), 47-97.

[15] S. Brin, L. Page, "The anatomy of a large-scale hypertextual Web-search engine", Proceedings of the 7 th World-Wide Web Conference (14-18 april, 1998, Brisbane, Australia), Brisbane, 1998, 107-117.

\section{В. М. Челноков}

Поступило

Межотраслевой научно-исследовательский институт

03.09.2007

"Интеграл"

Исправленный вариант

\section{В. Л. Зефирова}

28.04 .2008

Московский государственный университет

им. М. В. Ломоносова

E-mail: victoria@smisc.net.ru 